\title{
Spatially controlled glycocalyx engineering for growth factor patterning in embryoid bodies
}

\author{
Matthew R. Naticchia, ${ }^{\ddagger[a]}$ Logan K. Laubach,,$\left[{ }^{[a]}\right.$ Daniel J. Honigfort, ${ }^{[a]}$ Sean C. Purcell,,$\left[{ }^{[a]}\right.$ \\ and Kamil Godula*[a]
}

Keywords: glycocalyx, glycomimetics, embryoid body, FGF2, gradient

\begin{abstract}
Growth factor (GF) patterning in stem cell spheroids, such as embryoid bodies (EBs), has been sought to guide their differentiation and organization into functional 3D tissue models and organoids. Current approaches relying on exposure of EBs to gradients of GFs suffer from poor molecular transport in the spheroid microenvironment and from high cost of production and low stability of recombinant GFs. We have developed an alternative method for establishing GF gradients in EBs utilizing stem cell surface engineering with membrane-targeting heparan sulfate-glycomimetic co-receptors for GFs. We have capitalized on the ability of amphiphilic lipid-functionalized glycopolymers with affinity for FGF2 to assemble into nanoscale vesicles with tunable dimensions and extracellular matrix penetrance. Upon size-dependent diffusion into $\mathrm{EBs}$, the vesicles fused with the plasma membranes of stem cells, giving rise to concentric gradients of cells with enhanced FGF2-binding. The extracellular matrix-assisted cell surface remodeling process described is the first example of spatially-targeted glycocalyx engineering in multicellular systems to control GF localization. The glycopolymer structure, vesicle dimensions, and remodeling conditions determine the level of FGF2 adhesion and gradient slope. The increased chemical and thermal stability of the synthetic glycomimetics and the tunability of their GF-binding profile, which is defined by their glycosylation and may be extended to other recombinant or endogenous morphogens beyond FGF2, further increase the versatility of this method.
\end{abstract}

$\$$ M.R.N. and L.K.L. contributed equally.

${ }^{[\mathrm{a}]}$ Department of Chemistry and Biochemistry and ${ }^{[b]}$ Glycobiology Research and Training Center, University of California San Diego, 9500 Gilman Drive, La Jolla, CA 92093-0358, USA

* Corresponding Author: kgodula@ucsd.edu 


\section{Introduction.}

Embryonic stem cells (ESCs) and induced pluripotent stem cells (iPSCs) have the capacity to differentiate into any cell type of the adult body. ${ }^{1}$ They provide an important tool to study the process of embryogenesis and organismal development ${ }^{2}$ and hold promise for a range of biomedical applications. ${ }^{3}$ A key challenge in realizing their full potential is in translating differentiation protocols established in $2 \mathrm{D}$ in vitro cultures to the highly complex 3D environments of tissues in vivo. ${ }^{4,5} 3 \mathrm{D}$ spheroids formed from ESCs and iPSCs, such as embryoid bodies (EB), ${ }^{6}$ offer a convenient laboratory system to bridge this gap. EBs have the capacity to undergo spontaneous self-patterning into ordered multicellular structures, ${ }^{7}$ however, harnessing this potential for reliable production of functional tissue and organ replacements will require achieving better spatial and temporal control over cell differentiation in these systems. ${ }^{8}$

Current methods for engineering the microenvironment and self-organization of stem cell spheroids focus primarily on directing the delivery, release and diffusion of differentiation cues, such as growth factors (GFs) and small molecule morphogens. ${ }^{9,10}$ For instance, spatial control over cell differentiation in EBs has been accomplished through their exposure to GF gradients in microfluidic devices ${ }^{11,12}$ or through encapsulation in hydrogels with controlled GF release (Fig 1A). ${ }^{13,14}$ The inherent challenge in these approaches is a restricted transport of morphogens into the spheroids through their outer shell composed of tightly associated cells and dense deposits of extracellular matrix (ECM). ${ }^{15,16}$ Imbedding of GFladen microparticles with stem cells during EB aggregation overcomes this challenge; however, it provides limited control over the localization of the GF source within the spheroid (Fig 1A). ${ }^{17,18}$

Here, we present an alternative chemical method for generating gradients of GFs in EBs, which takes advantage of the restricted macromolecular transport across the spheroid boundary (Fig 1B). Our approach utilizes lipid-modified glycopolymers (GPs) with tunable size and affinity for GFs, which insert directly into the plasma membranes of ESCs and promote GF adhesion. Pre-assembly of the amphiphilic glycomimetics into nanoscale glycopolymer vesicles (GPVs) with defined dimensions enables tuning of their diffusion into the EBs, upon which they undergo fusion with the plasma membranes of ESCs to produce gradients of cells with enhanced GF-affinity (Fig 1B).

\section{Results and discussion.}

The glycomimetics are inspired by heparan sulfate (HS) glycosaminoglycans (GAGs), which are sulfated polysaccharide co-receptors for GFs on surfaces of cells and regulators of their activity, ${ }^{19}$ including during stem cell differentiation. ${ }^{20}$ Synthetic GAG mimetics have emerged as readily accessible biomaterials with capacity to bind and regulate the activity of a range of HS-binding proteins. Comprising linear polymeric scaffolds decorated with variously sulfated synthetic disaccharides representing the elementary structural motif of HS, soluble HS-mimetic glycopolymers have been shown to attenuate B cell chemotaxis induced by the chemokine RANTES ${ }^{21}$ or inhibit breast cell metastasis in mouse models by blocking the activity of matrix heparinases. ${ }^{22}$ We have developed HS-mimetic polymers with affinity for various GFs comprising enzymatically derived HS disaccharides. ${ }^{23,24}$ Endowing the polymers with phospholipid tails allowed for their targeting to the plasma membranes of ECSs. There, the materials served as functional surrogates for native GAGs, activated signaling by FGF2 and BMP4, and promoted neural and mesodermal differentiation, respectively. We envisioned that the phospholipids, which allows for insertion of the HS mimetics into the plasma membranes of cells, also give the GPs amphiphilic character and should promote their assembly into GPVs (Fig 1B). Polymer length as well as the size and charge of their glycans should allow for tuning of GPV size and penetrance into the EBs (Fig 1B).

To test this hypothesis, we generated a panel of GPs ranging in size from short ( $\mathbf{S}$, degree of polymerization, DP 40) to medium (M, DP 125) and long (L, DP 300) and glycosylated either with the 2,6-Odisulfated HS-derived disaccharide, $\triangle \mathrm{UA2S}-$ GlcNAc6S (D2A6), or the monosaccharides $N$-acetylglucosamine (GlcNAc) and its 6-O-sulfated derivative, GlcNAc-6S (Fig 2A). The GPs were derived from a Boc-protected poly(3-N-methylaminooxypropyl) acrylamide precursors (P1) generated by RAFT ${ }^{25}$ 
polymerization of monomer $\mathbf{1}$ in the presence of a dipalmitoyl phospholipid-containing chain transfer agent CTA and the radical initiator, AIBN (Fig 2A). ${ }^{23}$ The RAFT process furnished precursors P1 with good control over molecular weight and dispersity (Đ), as determined by size exclusion chromatography (SEC, Figs 2B and S1).

The polymeric precursors P1 were elaborated in four steps to produce glycopolymers GP labeled with AlexaFluor488 (AF488) for visualization (Fig 2A). First, the trithiocarbonate chain end groups in polymers P1 were cleaved with $n$-butylamine to release a reactive thiol, which was capped immediately with AF488-maleimide. The protective Boc groups were then removed by treatment with trimethylsilyl chloride in phenol to yield polymers $\mathbf{P 2}$ with exposed $N$-methylaminooxy side chains for subsequent ligation of reducing glycans. ${ }^{26}$ The extent of polymer labeling $\left(f_{A F 488}\right)$ was determined by UV-VIS at this stage to be $\sim 0.7$ - 0.8 fluorophores per polymer chain (Table S3). Finally, heating of intermediates $\mathbf{P 2}$ with the mono- and disaccharides at $50{ }^{\circ} \mathrm{C}$ under acidic conditions (acetate buffer, $\mathrm{pH}=4.5$ ), followed by size filtration yielded the desired GPs (Fig 2C). The fraction of glycosylated side chains was determined for each glycopolymer by ${ }^{1} \mathrm{H}$ NMR spectroscopy and ranged from $\sim 40-50 \%$ for the charged glycans, D2A6 and GlcNAc-6S, to $\sim 50-60 \%$ for the neutral monosaccharide, GlcNAc (Fig 2C and Table S4).

We evaluated the affinity of the negatively charged HS-mimetic glycopolymers for FGF2, which induces ECSs toward neural commitment. ${ }^{20}$ Using ELISA with surface-immobilized FGF2 (Fig S3), we measured strong binding for polymers GP-D2A6 carrying the 2,6- $O$-disulfated HS disaccharides $\left(K_{\text {d,app }}=2-7 \mathrm{nM}\right)$. The observed affinity was comparable to that of heparin $\left(K_{\mathrm{d} \text {,app }}=6 \mathrm{nM}\right)$, which is a highly sulfated form HS. The monosaccharide-modified polymers GP-GIcNAc-6S showed no measurable FGF2 binding activity (Fig S3).

Dynamic light scattering (DLS) analysis of the GP solutions in PBS buffer $(\mathrm{pH}=7.4)$ confirmed their assembly into GPVs with sizes of $\sim 40-150 \mathrm{~nm}$ (Figs 3 and S4). The molecular weight (Mw) of the polymers scaled linearly with their length (DP). The steepest rise in Mw was observed for the disaccharide containing polymers, GP-D2A6, followed by the monosaccharide polymers GP-GlcNAc-6S and GPGIcNAc (Fig 3A). Accordingly, the short (S), medium (M), and long (L) glycopolymers GP-D2A6 afforded the largest GPVs (Figs 3B and S4, Table S5) with diameters $\left(d_{\mathbf{G P V}}\right)$ of $44 \pm 1,106 \pm 9$, and $139 \pm$ $12 \mathrm{~nm}$, respectively (Fig 3B and 3C). The monosaccharide analogs GP-GlcNAc-6S and GP-GIcNAc gave GPVs of approximately equal sizes for each polymer length: $42 \pm 8$ and $36 \pm 1 \mathrm{~nm}(\mathbf{S}), 64 \pm 5$ and $65 \pm 4 \mathrm{~nm}(\mathbf{M})$, and $74 \pm 13$ and $84 \pm 24 \mathrm{~nm}(\mathbf{L})$. Polymers without the lipids remained dissolved and did not form GPVs. Our DLS data indicate that the GPV size is determined primarily by the DP and Mw of the glycopolymers but not their charge, which was reflected in the zeta potential of the vesicles (Fig 3D, Table S5).

The single short hydrophobic dipalmitoyl lipid tail attached to the much more extended hydrophilic glycosylated segment of the GPs is likely to drive the formation of vesicles rather than micelles, ${ }^{27}$ with the latter expected to produce much smaller particle sizes than those observed by DLS. The GPVs, which form during the glycopolymer assembly step, remain stable in solution over a range of concentrations $(0.1$ - $10.0 \mu \mathrm{M}$ ) for several days. The GPVs can be disrupted by the addition a detergent, such as TritonX-100 (Fig S4E). Irreversible aggregation of the GPVs was observed after freezing, drying, or prolonged storage in solution.

We envisioned that differential size-dependent diffusion of the GPVs through the ECM network of EBs, followed by their fusion with ESC membranes, would establish gradients of HS mimetic-remodeled stem cells with increasing FGF2 affinity (Fig 1B). To eliminate background FGF2 binding from endogenous HS glycans on ESCs, we used mutant cells missing the gene, exostosin-1 (Ext1), which encodes for a glycosyltransferase involved in HS chain polymerization. ${ }^{19}$ Unable to produce functional HS, the $\operatorname{Ext}^{-/-}$ ESCs lack the ability to bind many HS-dependent GFs, including FGF2. ${ }^{28}$ 
Since HS also contributes to cell adhesion and deposition of matrix proteins (e.g., fibronectin), ${ }^{29}$ we first analyzed the effects of the Ext1 gene deletion on EB formation and ECM organization. Wild type (wt) E14 and Ext $1^{-/-}$ESCs were aggregated into EBs under embryonic conditions in the presence of LIF using the hanging drop method (Fig 4A). ${ }^{30}$ After 2 days, both cell lines formed EB spheroids with normal morphology and sizes of $\sim 100-200 \mu \mathrm{m}$ (Figs 4B and S5). Scanning electron microscopy (SEM) imaging confirmed the formation of ECM deposits on the surface of EBs from both cell lines (Figs 4C and S6). Immunohistological analysis of the EBs confirmed the lack of HS expression by Ext1 $1^{-/-}$ESCs, which resulted in accumulation of fibronectin toward the EB periphery but had no observable effect on collagen deposition (Fig 4D and Fig S7). The ability of Ext1 $1^{-/}$ESCs to form EBs and deposit ECM, we reasoned, should influence GPV penetrance into the spheroids.

Next, we set to test whether the GPVs can remodel the glycocalyx of ESCs in a gradient pattern after diffusion into the EBs (Fig 5). In addition to their size, EB penetrance of the GPVs is likely to be determined by their concentration in the media and the duration of treatment. We examined these parameters by first incubating Day $2 \mathrm{Ext}^{-/-}$ESC EBs with $0.1,1.0$ and $3.0 \mu \mathrm{M}$ fluorescently-labeled GP-M/GIcNAc6S in serum-free media. After 2 hrs at $37^{\circ} \mathrm{C}$, the EBs were washed to remove excess GPVs, fixed, and imaged by confocal microscopy to assess glycopolymer distribution.

Fluorescence images of cross-sections at the EB midpoint showed robust and uniform labeling with 3.0 $\mu \mathrm{M}$ GP-M/GIcNAc-6S (Fig S8). Dilution of the polymer resulted in overall lower levels of cell labeling and polymer accumulation toward the EB surface. By shortening the incubation time at the highest polymer concentration $(3.0 \mu \mathrm{M})$ to $1 \mathrm{hr}$, we retained robust cell labeling while also establishing a concentric polymer gradient across the spheroid (Fig S9). Treatment of the EBs with glycopolymers GP-GIcNAc$\mathbf{6 S}$ of all three lengths under the optimized conditions $\left(3.0 \mu \mathrm{M}, 1 \mathrm{hr}, 37^{\circ} \mathrm{C}\right)$ resulted in differential diffusion of their GPVs into EBs according to their size (Figs 5A and S10). We observed a transition from complete GPV penetrance for the shortest polymer GP-S/GIcNAc-6S (DP $\sim 40, d_{\mathbf{G P V}}=42 \mathrm{~nm}$ ) to increasing outer cell layer accumulation as the polymer size and GPV diameter increased (cf. GPL/GIcNAc-6S, DP 300, $\left.d_{\mathbf{G P V}}=74 \mathrm{~nm}\right)$.

We sought to develop a set of quantitative measures to assess GPVs diffusion into the EBs and to characterize GP gradients after ESC remodeling. We collected fluorescence micrographs of EB cross-sections at their widest point and analyzed fluorescence intensity from the polymers $\left(F_{488}\right)$ along a line passing through the EB center (Fig 5A). We then plotted the normalized intensities against distance from EB center (Fig 5A, green). The area under the curve provided a quantitative measure of GPV penetrance into the EB (Fig 5B, bars). To address signal variability arising from local microheterogeneities within the EB structures, we performed bin analysis of the normalized line fluorescence intensity and generated intensity histograms (Fig 5A, bars), where each bar represents mean pixel intensity over a $10 \mu \mathrm{m}$ bin.

To evaluate how rapidly the gradient of GP-remodeled cells changes with GPV diffusion into EBs, we plotted the absolute values of line fluorescence intensity change $\left(F_{488}^{\prime}\right)$ against distance from EB center (Fig 5B, line graph). In agreement with our visual assessment of cross-section images, we observed the slowest change in fluorescence intensity for GP-S/GIcNAc-6S, consistent with its highest penetrance and uniform distribution across the EB. The rate of gradient change increased with polymer length and GPV size. The neutral polymer GP-L-GIcNAc did not exhibit enhanced diffusion compared to the sulfated analogs GlcNAc-6S analog, indicating that GPV diameter rather than charge determines EB penetrance (Figs S11 and S12).

The polymer fluorescence after EB remodeling was localized to the ESC surface, indicating insertion of the GPs into the cell membranes via their lipid tails (Fig S10D). To confirm membrane tethering, EBs treated with GP-S/GIcNAc-6S and GP-L/GIcNAc-6S were dissociated into single cells by Accutase, 
washed to remove adsorbed GPVs, and analyzed by flow cytometry (Fig S13). Both polymers remained associated with the cell surface and the population of labeled cells increased with higher EB penetrance of the GPs, consistent with fluorescence image analysis (Fig 5B).

Focusing on our aim to exploit the size-limited diffusion of GPVs in the EB environment to engineer GF affinity gradients, we performed remodeling experiments with the FGF2-binding HS mimetics, GPD2A6. We have previously shown that installation of glycopolymers carrying the D2A6 disaccharide at the surface of Ext1 $1^{-/}$ESCs restored FGF2 binding and signaling activity, ${ }^{23}$ thus providing functional surrogates for cell-surface HS. While the $w t$ E14 ESCs express endogenous HS structures that produce uniform FGF2 binding across the EB, the $\mathrm{Ext1}^{-/-} \mathrm{ESCs} \mathrm{HS}$ mutants lack affinity for FGF2 and provide a clean slate for the pattering of the GF (Fig 5C).

Treatment of Day 2 Ext $1^{-/-}$ESC EBs with GP-D2A6 polymers of all three lengths under the optimized conditions $\left(3.0 \mu \mathrm{M}, 1 \mathrm{hr}, 37^{\circ} \mathrm{C}\right)$ resulted in patterns of remodeled cells analogous to those observed for the model monosaccharide polymers GP-GIcNAc-6S (Figs 5D and 5A, green). The shortest polymers GP-S/D2A6 $\left(d_{\mathbf{G P V}}=44 \mathrm{~nm}\right)$ gave full coverage of the EB interior similar to its monosaccharide derivative GP-S/GIcNAc-6S which forms GPVs of comparable size $\left(d_{\mathbf{G P V}}=42 \mathrm{~nm}\right)$. By contrast, the medium-sized polymer GP-M/D2A6 $\left(d_{\mathbf{G P V}}=106 \mathrm{~nm}\right)$ exhibited lower EB penetrance and generated a steeper fluorescence gradient compared to GP-M/GlcNAc-6S (with $d_{\mathbf{G P V}}=64 \mathrm{~nm}$ ). The same trend continued for the pair of longest polymers, GP-L/D2A6 $\left(d_{\mathbf{G P V}}=139 \mathrm{~nm}\right)$ and GP-L/GIcNAc-6S $\left(d_{\mathbf{G P V}}=74 \mathrm{~nm}\right)$. Our observations are consistent with GP diffusion into the EB spheroids being determined primarily by the size of their GPVs independent of the structure and charge state of their glycans, which provides an element of predictability and tunability.

The patterns of cell-surface anchored HS-mimetics, GP-D2A6, in turn, defined the distribution of FGF2 adhesion sites in the Ext1 $1^{-/}$ESC EBs, which were visualized by incubation with recombinant FGF2 followed by immunostaining (Fig 5D, red, and Figs S14 and S15). No FGF2 signal was observed in EBs treated with polymers GP-GIcNAc-6S (Fig S16), confirming that the GF binding is glycan dependent and the display of the HS mimetics GP-D2A6 on the surface of ESCs is required to constrain FGF2 localization.

\section{Conclusions.}

We have developed a novel, facile method for generating GF gradients in multicellular spheroids using cell membrane engineering with synthetic GF co-receptors. By exploiting the assembly of amphiphilic glycopolymers into nanoscale vesicles with tunable diameters, we were able to control their diffusion into EB spheroids. Upon entry, the GPVs fused with the membranes of nearby stem cells and introduced new adhesion sites for FGF2 at their surface. By using Ext $1^{-/-}$ESCs lacking endogenous HS, we were able to limit FGF2 adhesion only to cells remodeled with appropriately glycosylated HS mimetics. The formation of vesicles and their fusion into the membranes of cells within EBs provides a unique delivery method for glycan-based receptors to the surface of cells in complex systems. In departure from existing methods for gradient patterning in 3D spheroids, which rely on the controlled diffusion and release of recombinant GFs, the current approach achieves GF patterning by tailoring their interactions at the cell surface and, thus, provides control over the activity of endogenous GFs. The cell surface engineering method can be extended to other types of cells and HS-dependent signaling molecules, and we expect its utility to increase further with the continuing improvements in HS mimetic design ${ }^{21,22}$ and with the emergence of new tools for the genetic ${ }^{31}$ and chemical ${ }^{32}$ manipulation of HS expression in living cells. Further, we expect that the discovery of assembly of amphiphilic glycomaterials into tunable and stable vesicles capable of direct fusion with cell membranes will open new modalities for glycocalyx engineering in increasingly complex biological systems. 


\section{Conflict of interest}

There are no conflicts to declare.

\section{Acknowledgment.}

The authors thank Dr. Ryan Porell (UCSD) and Dr. Mia Huang (TSRI) for valuable discussions and their insights on experimental methods and data analysis. We also thank Dr. Cathy Merry (University of Nottingham) for providing us with ESCs. We wish to acknowledge the UCSD Microscopy Core Facility (via NINDS P30 Grant: P30NS047101) for assistance with fluorescence confocal imaging. SEM work was performed at the San Diego Nanotechnology Infrastructure (SDNI) of UCSD, a member of the National Nanotechnology Coordinated Infra-structure (NNCI) supported by NSF grant ECCS-1542148. We thank UCSD Mouse Phenotyping Core at the GRTC (supported via NHLBI grant P01HL107150) for assistance with histological analysis. This work was supported by NIH Director's New Innovator Award 1DP2HD087954-01 via NICHD. M.R.N. and S.C.P. were supported by GAANN fellowships (U.S. Dept. Of Education, P200A150251). K.G. was supported in part by the Alfred P. Sloan Foundation (FG-20179094) and the Research Corporation for Science Advancement via the Cottrell Scholar Award (24119). 


\section{References.}

1. Moradi, S.; Mahdizadeh, H.; Šarić, T.; et al. Research and therapy with induced pluripotent stem cells (iPSCs): social, legal, and ethical considerations. Stem Cell Res Ther. 2019, 10, 341.

2. Dvash, T.; Ben-Yosef, D.; Eiges, R. Human embryonic stem cells as a powerful tool for studying human embryogenesis. Pediatr Res. 2006, 60, 111-117.

3. Bragança, J.; Lopes, J. A.; Mendes-Silva, L.; Almeida Santos, J. M. Induced pluripotent stem cells, a giant leap for mankind therapeutic applications. World J Stem Cells. 2019, 11, 421-430.

4. Antoni, D.; Burckel, H.; Josset, E.; Noel, G. Three-dimensional cell culture: a breakthrough in vivo. Int J Mol Sci. 2015, 16, 5517-5527.

5. Lee, J.; Cuddihy, M. J.; Kotov NA. Three-dimensional cell culture matrices: state of the art. Tissue Eng Part B Rev. 2008, 14, 61-86.

6. Bratt-Leal, A. M.; Carpenedo, R. L.; McDevitt, T. C. Engineering the embryoid body microenvironment to direct embryonic stem cell differentiation. Biotechnol Prog. 2009, 25, 43-51.

7. Ruiz, S. A.; Chen, C. S. Emergence of patterned stem cell differentiation within multicellular structures. Stem Cells. 2008, 26, 2921-2927.

8. Sasai, Y. Next-generation regenerative medicine: organogenesis from stem cells in 3D culture. Cell Stem Cell. 2013, 12, 520-530.

9. Brassard, J. A.; Lutolf, M. P. Engineering Stem Cell Self-organization to Build Better Organoids. Cell Stem Cell. 2019, 24, 860-876.

10. Samal, P.; van Blitterswijk, C.; Truckenmüller, R.; Giselbrecht, S. Grow with the Flow: When Morphogenesis Meets Microfluidics. Adv Mater. 2019, 31, e1805764.

11. Torisawa, Y. S.; Chueh, B. H.; Huh, D.; et al. Efficient formation of uniform-sized embryoid bodies using a compartmentalized microchannel device. Lab Chip. 2007, 7, 770-776.

12. Fung, W. T.; Beyzavi, A.; Abgrall, P.; Nguyen, N. T.; Li, H. Y. Microfluidic platform for controlling the differentiation of embryoid bodies. Lab Chip. 2009, 9, 2591-2595.

13. Gerecht, S.; Burdick, J. A.; Ferreira, L. S.; Townsend, S. A.; Langer, R.; Vunjak-Novakovic, G. Hyaluronic acid hydrogel for controlled self-renewal and differentiation of human embryonic stem cells. Proc. Natl. Acad. Sci.; U S A. 2007, 104, 11298-11303.

14. Mosiewicz, K. A.; Kolb, L.; van der Vlies, A. J.; et al. In situ cell manipulation through enzymatic hydrogel photopatterning. Nat Mater. 2013, 12, 1072-1078.

15. Van Winkle, A. P.; Gates, I. D.; Kallos, M. S. Mass transfer limitations in embryoid bodies during human embryonic stem cell differentiation. Cells Tissues Organs. 2012, 196, 34-47.

16. Carpenedo, R. L.; Bratt-Leal, A. M.; Marklein, R. A.; et al. Homogeneous and organized differentiation within embryoid bodies induced by microsphere-mediated delivery of small molecules. Biomaterials. 2009, 30, 2507-2515.

17. Ferreira, L.; Squier, T.; Park, H.; Choe, H.; Kohane, D. S.; Langer, R. Human embryoid bodies containing nano- and microparticulate delivery vehicles. Adv Mater. 2008, 20, 2285-2291.

18. Bratt-Leal, A. M.; Nguyen, A. H.; Hammersmith, K. A.; Singh, A.; McDevitt, T. C. A microparticle approach to morphogen delivery within pluripotent stem cell aggregates. Biomaterials. 2013, 34, 7227-7235.

19. Xu, D.; Esko, J. D. Demystifying heparan sulfate-protein Interactions. Annu. Rev. Biochem. 2014, 83, 129-157.

20. Kraushaar, D. C.; Dalton, S.; Wang, L. Heparan sulfate: a key regulator of embryonic stem cell fate. Biol Chem. 2013, 394, 741-751.

21. Sheng, G. J.; Oh, Y. I.; Chang, S. K.; Hsieh-Wilson, L. C. Tunable heparan sulfate mimetics for modulating chemokine activity. J. Am. Chem. Soc. 2013, 135, 10898-10901.

22. Loka, R. S.; Sletten, E. T.; Barash, U.; Vlodavsky, I.; Nguyen, H. M. Specific Inhibition of Heparanase by a Glycopolymer with Well-Defined Sulfation Pattern Prevents Breast Cancer Metastasis in Mice. ACS Appl Mater Interfaces. 2019, 11, 244-254. 
23. Huang, M. L.; Smith, R. A.; Trieger, G. W.; Godula K. Glycocalyx remodeling with proteoglycan mimetics promotes neural specification in embryonic stem cells. J. Am. Chem. Soc. 2014, 136, 1056510568.

24. Lawrence R, Lu H, Rosenberg RD, Esko JD, Zhang L. Disaccharide structure code for the easy representation of constituent oligosaccharides from glycosaminoglycans. Nat Methods. 2008, 5, 291-292.

25. Moad, G.; Rizzardo, E.; Thang, S. H. Living Radical Polymerization by the RAFT Process - A Second Update. Aust. J. Chem. 2009, 62, 1402-1472.

26. F. Peri, P. Dumy, M. Mutter, Chemo- and stereoselective glycosylation of hydroxylamino derivatives: A versatile approach to glycoconjugates. Tetrahedron. 1998, 54, 12269-12278.

27. Lombardo, D.; Kiselev, M. A.; Magazù, S.; Calandra, P. Amphiphiles Self-Assembly: Basic Concepts and Future Perspectives of Supramolecular Approaches. Adv. Cond. Matter Phys. 2015, e151683.

28. Kraushaar, D. C.; Yamaguchi, Y.; Wang, L. Heparan sulfate is required for embryonic stem cells to exit from self-renewal. J. Biol. Chem. 2010, 285, 5907-5916.

29. Raitman, I.; Huang, M. L.; Williams, S. A.; Friedman, B.; Godula, K.; Schwarzbauer, J. E. Heparinfibronectin interactions in the development of extracellular matrix insolubility. Matrix Biol. 2018, 67, 107-122.

30. Koike, M.; Sakaki, S.; Amano, Y.; Kurosawa, H. Characterization of embryoid bodies of mouse embryonic stem cells formed under various culture conditions and estimation of differentiation status of such bodies. J. Biosci. Bioeng. 2007, 104, 294-299.

31. (a) Chen, Y. H.; Narimatsu, Y.; Clausen, T. M.; et al. The GAGOme: a cell-based library of displayed glycosaminoglycans. Nat. Methods 2018, 15, 881-888. (b) Qiu, H.; Shi, S.; Yue, J.; et al. A mutantcell library for systematic analysis of heparan sulfate structure-function relationships. Nat. Methods. 2018, 15, 889-899.

32. Chua, J. S.; Kuberan, B. Synthetic Xylosides: Probing the Glycosaminoglycan Biosynthetic Machinery for Biomedical Applications. Acc. Chem. Res. 2017, 50, 2693-2705. 
Figures:

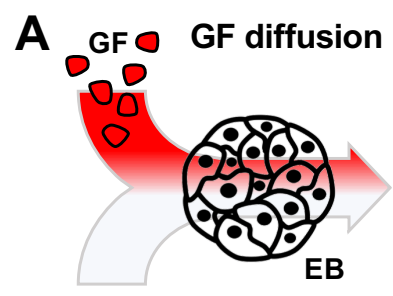

microfluidics
GF release within cell spheroid

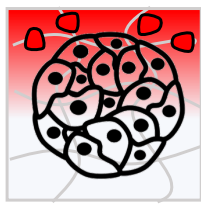

engineered matrix

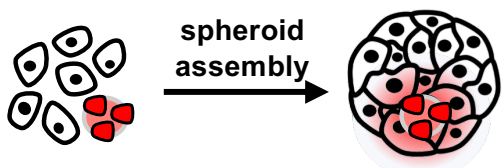

imbedded GF source

B GF patterning via extracellular matrix-assisted glycocalyx engineering

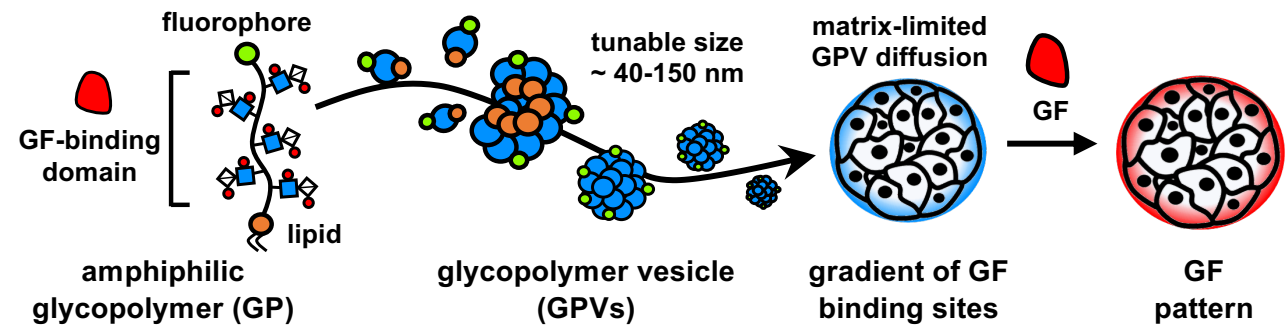

Figure 1. Growth factor (GF) gradient engineering in stem cell spheroids. A) Prior methods utilize exposure of spheroids to GF gradients in microfluidic devices and engineered hydrogels or through imbedding of GF-laden microparticles in the spheroid core. B) The present approach uses cell surface engineering with glycomimetics to tailor stem cell interactions with GFs. Amphiphilic GF-binding glycopolymers (GPs) assemble into glycopolymeric vesicles (GPVs) with tunable size and extracellular matrix penetrance. Upon entry into the spheroid, the GPVs fuse with cell membranes, forming gradients of stem cells with enhanced GF affinity. 
A<smiles>C=CC(=O)NON(C)C(=O)OC(C)(C)C</smiles>

1

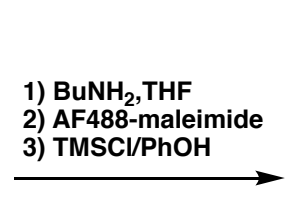<smiles>CN(ON(C)OC(C)(C)C)OC(C)(C)C</smiles>

O CTA

AIBN, dioxane, $65^{\circ} \mathrm{C}$

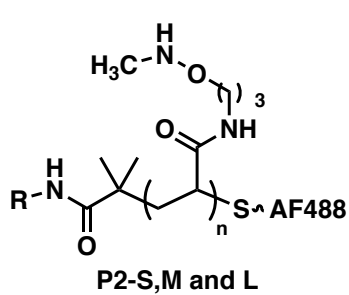

$\mathrm{R}=-\left(\mathrm{CH}_{2}\right)_{2} \mathrm{O}$

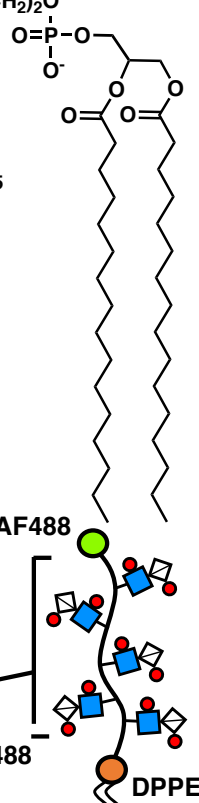
HS mimetic glycopolymers (GP-S,M and L)

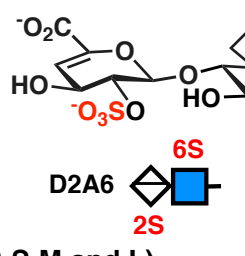<smiles>[R]NC(=O)C(C)(C)CC(CC(C)(C)C(=O)O)C(=O)SC(=S)NC</smiles>

P1: $S(D P=40)$ $M(D P=125)$ $L(D P=300)$ glycan (1.1 equiv)
NaOAc, pH $4.5,50^{\circ} \mathrm{C}$ NaOAc, pH $4.5,50{ }^{\circ} \mathrm{C}$

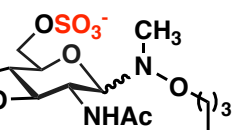

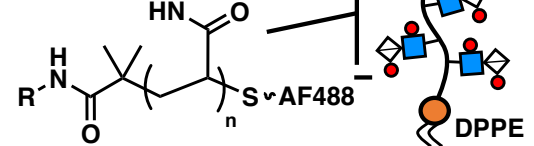

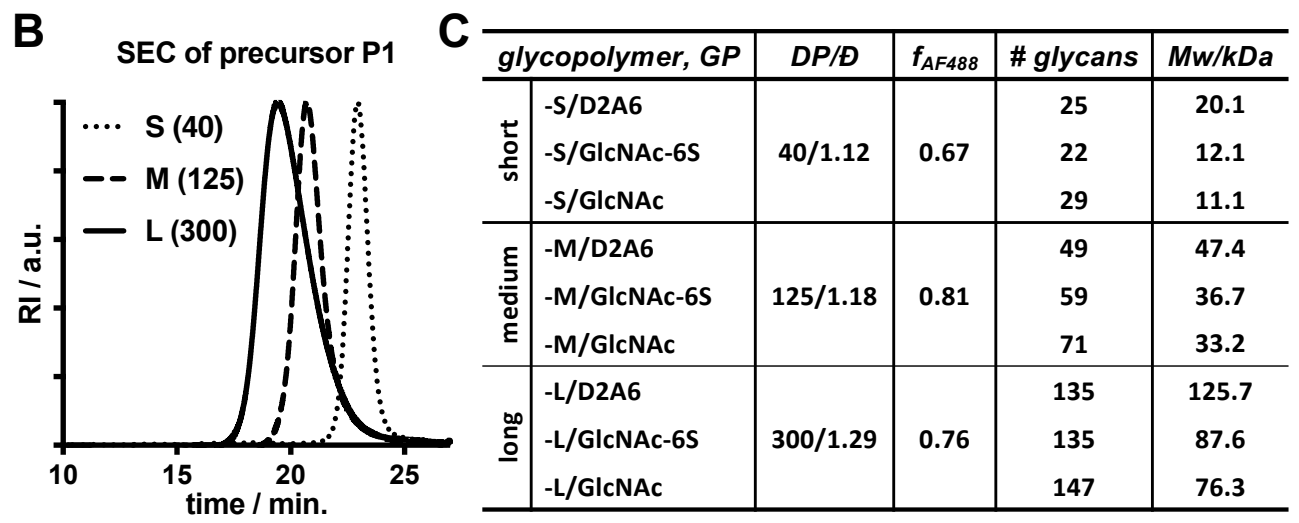

Figure 2. Synthesis and characterization of lipid-terminated HS-mimetic glycopolymers, GP. A) Short (S), medium (M), and long (L) GPs were assembled from a RAFT-derived polymeric precursor P1. B) Size exclusion chromatography (SEC) traces of P1-S, $\mathbf{M}$ and $\mathbf{L}$ ( $\mathrm{DP}=40,125$ and 300, respectively). C) Structural characteristics of GPs. $\mathrm{DP}=$ degree of polymerization, $\mathrm{\Xi}=$ dispersity, $f_{\mathrm{AF} 488}=$ fraction of AlexaFluor 488 (AF488)-labeled chains. 
A Mw of glycopolymers (GP)

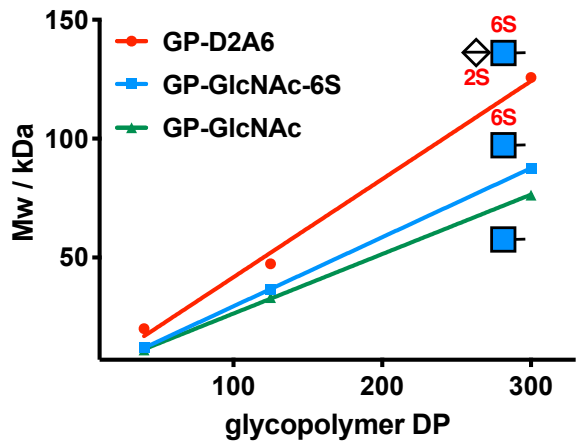

C

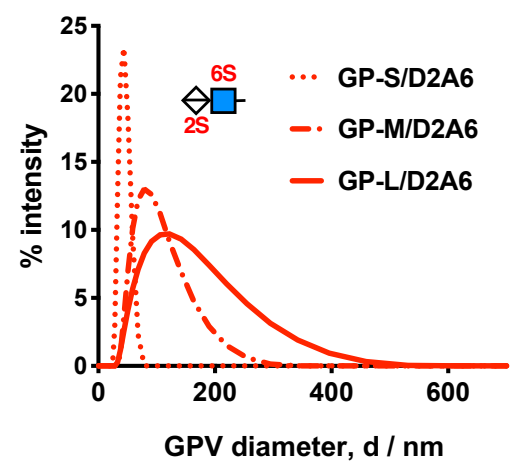

B glycopolymer vesicle (GPV) size

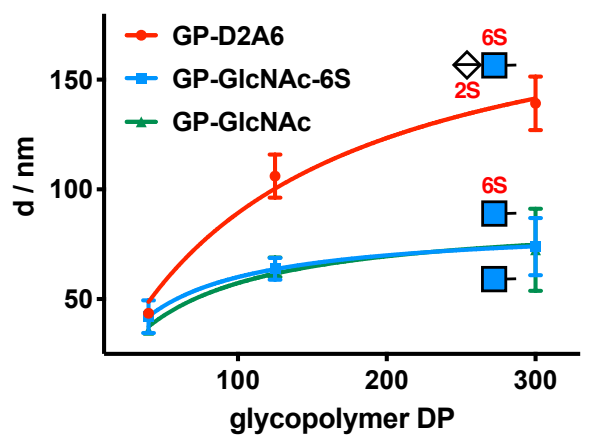

zeta potential (GPV-L)

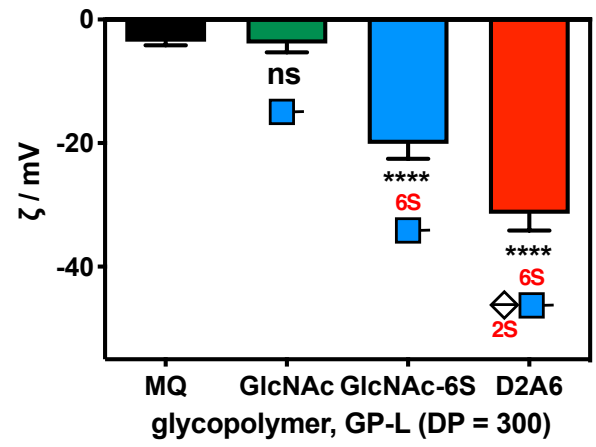

Figure 3. Assembly of amphiphilic HS-mimetic glycopolymers (GPs) into glycopolymeric vesicles (GPVs) with tunable diameter. A) Mw of glycopolymers GP increased proportionally with polymer DP and glycosylation. B) Short (S), medium (M), and long (L) GPs $(3 \mu \mathrm{M})$ assembled in water into GPVs. Polymer Mw, but not charge, determined GPV diameter. C) Size distributions for GPVs formed by polymers GP-D2A6. D) Zeta potential of GPVs (shown for glycopolymers GP-L), MQ = MilliQ water, $* * * * p<0.001$. 


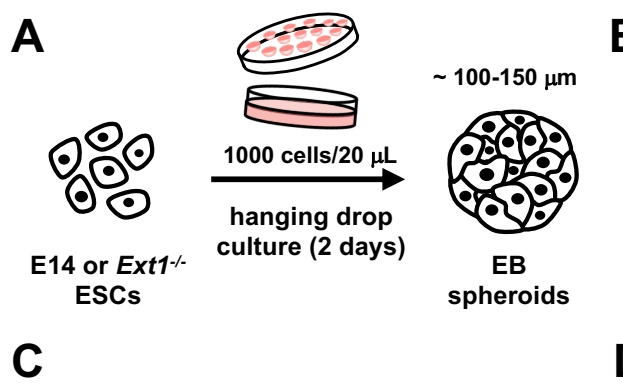

B

Day 2 embryoid bodies (EBs)
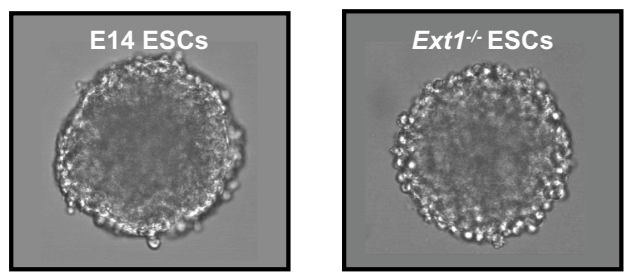

D
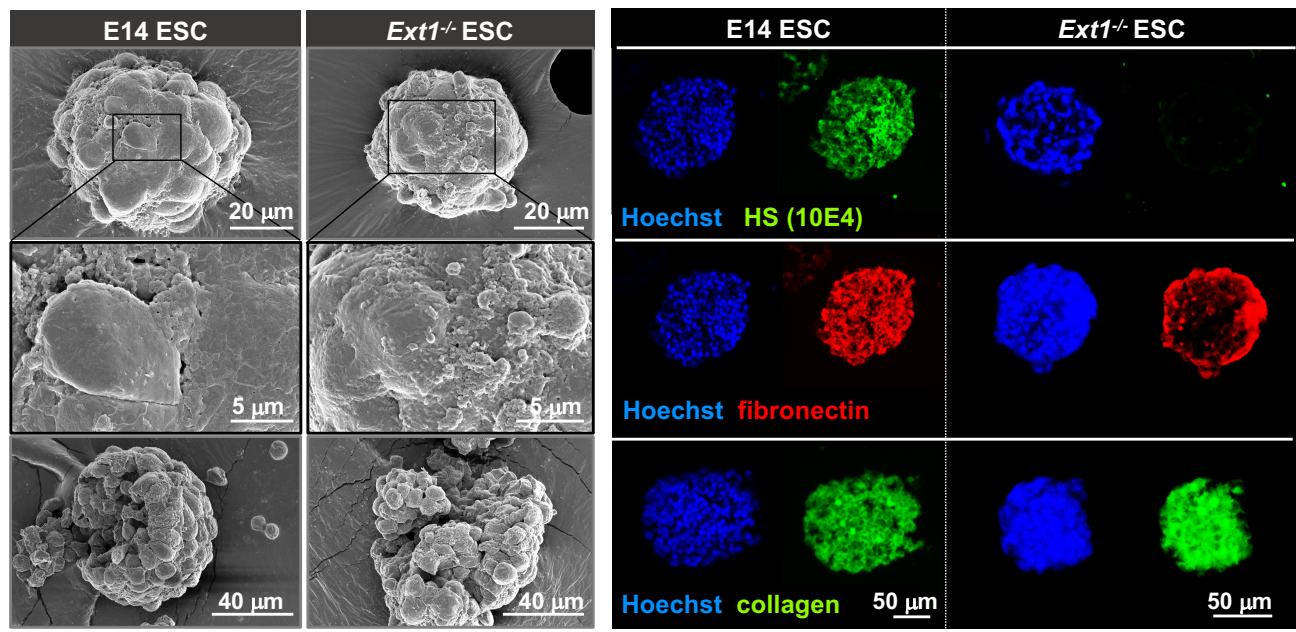

Figure 4. Formation and characterization of embryoid bodies (EBs). A) E14 and Ext1 ${ }^{-/}$ESCs were aggregated in hanging drops in the presence of LIF for 2 days. B) Optical microscopy confirmed normal EB formation. C) SEM images show similar level of ECM deposition on the surfaces of EBs from both cell lines. D) Immunostaining with anti-HS antibody, 10E4, confirmed lack of HS expression by Ext $1^{-/}$ESCs. Loss of HS led to accumulation of fibronectin toward the outer regions of the EBs but had no effect on collagen deposition (blue $=$ Hoechst 33342 nuclear stain). 

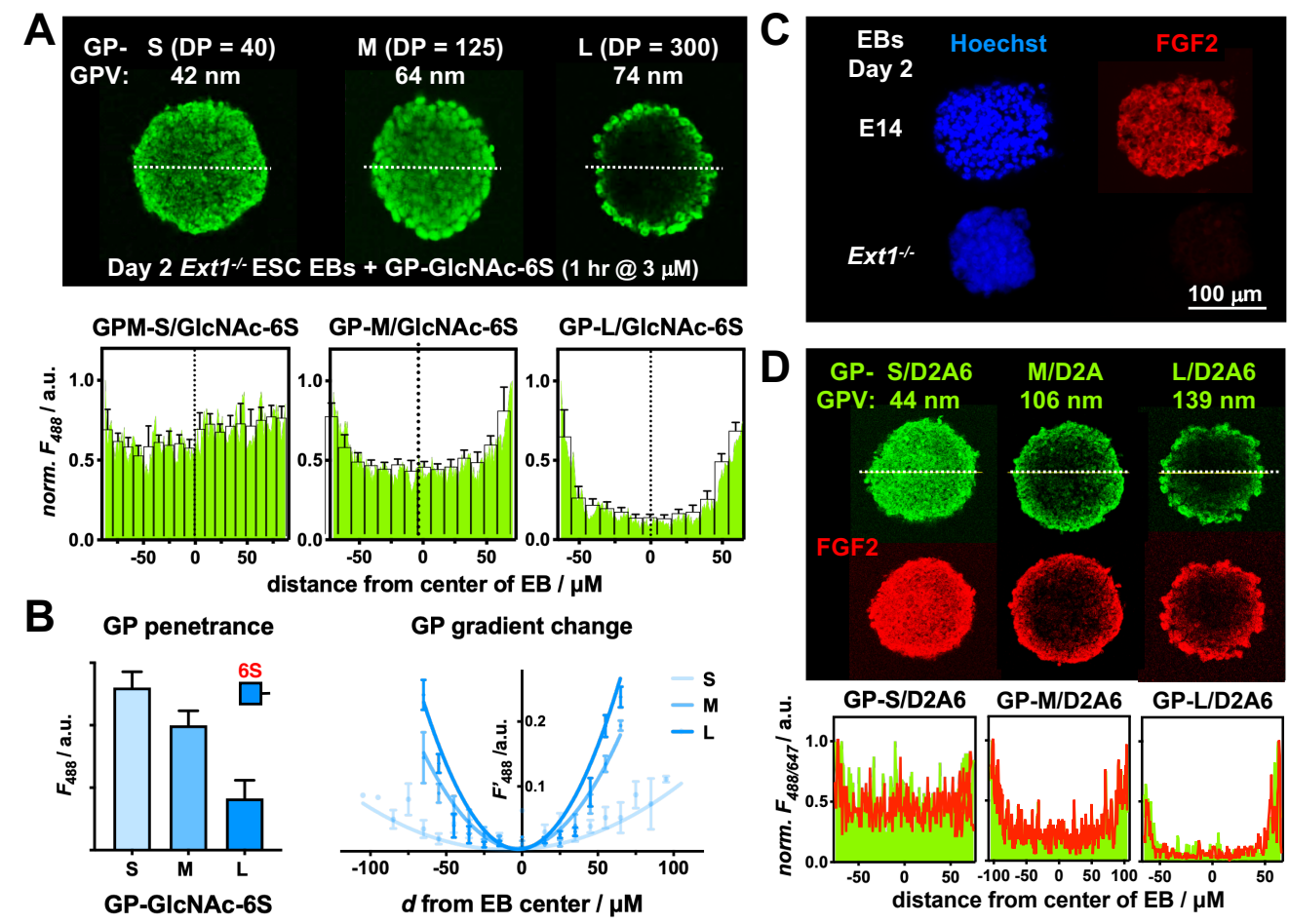

Figure 5. Engineering FGF2 affinity gradients in EB spheroids. A) Top: Fluorescence confocal micrographs of EB cross-sections at midpoint after treatment with short (S), medium (M), and long (L) AF488labeled glycopolymers GP-GIcNAc-6S. Bottom: Normalized fluorescence intensity (green) and bin histogram analysis (bars, 1 bin $=10 \mu \mathrm{m})$ of AF488 signal $\left(F_{488}\right)$ along a line passing through EB center. B) Total fluorescence intensity $\left(F_{488}\right)$ and rate of intensity change with distance from EB center $\left(F^{\prime}{ }_{488}\right)$. C) FGF2 (red) binds to $w t$ E14 but not Ext $1^{-/-}$ESCs in EBs. D) Fluorescence micrographs and normalized line fluorescence for FGF2 ( $r e d$ ) binding to EBs remodeled with glycopolymers GP-D2A6 (green). 